\title{
Empleo informal. Evolución teórica y criterios para su estimación derivados de la Organización Internacional del Trabajo (OIT)*
}

\section{Informal employment. Theoretical evolution and criteria for its estimation derived from the International Labour Organization (ILO)}

\section{Resumen}

Talina Contreras Dávila

Este artículo tiene el objetivo de contribuir al análisis teórico del empleo informal y a la definición de criterios para su estimación. Las discusiones sobre la informalidad del trabajo y las propuestas para su medición en América Latina iniciadas en la década de los setenta se han modificado y extendido conforme se acentúan la flexibilidad y la precariedad laboral. La Organización Internacional del Trabajo (OIT) ha estudiado el concepto hasta proporcionar nuevos elementos para su comprensión y medición tanto en países desarrollados como en desarrollo. Aquí se analizan los primeros enfoques sobre el sector informal y la evolución teórico-estadística de la OIT para revelar dos rutas de medición del empleo informal que incluyen al empleo asalariado como categoría esencial del mercado de trabajo. Concluye que el concepto puede ser operativo para analizar y estimar las condiciones de ocupación en economías con distintos niveles de desarrollo.

\section{Palabras clave}

Empleo informal, sector informal, Organización Internacional del Trabajo.

Códigos de clasificación JEL: J21, J81, J82.

\begin{abstract}
This article aims to contribute to the theoretical analysis of informal employment and to the definition of criteria for its estimation. The discussions on the informality of work and the proposals for its measurement in Latin America initiated in the 1970s have been modified and extended as labor flexibility and precariousness have become more pronounced. The International Labour Organization (ILO) has studied the concept to provide new elements for understanding and measurement in both developed and developing countries. Here, the first perspectives on the informal sector and the theoretical-statistical evolution of the ILO are analyzed in order to reveal two routes for measuring informal employment that include wage employment as an essential category of the labour market. The paper concludes that the concept can be operational for analysing and estimating employment conditions in economies with different levels of development.
\end{abstract}

\section{Keywords}

Informal employment, informal sector, International Labour Organization.

* Este artículo es derivado de la tesis doctoral de la autora, titulada "El empleo informal del colectivo latinoamericano con más residentes en España ante la crisis (una aproximación a su medición, 2005-2014)”, Universidad Complutense de Madrid.

$1 \quad$ Universidad de Barcelona. Correo electrónico: talinacontreras@gmail.com Código ORCID: 0000-0002-1182-9119. 


\section{Introducción}

El régimen de producción actual ha transformado los mercados de trabajo en todo el mundo. Las exigencias de una demanda que cambia constantemente han llevado a rápidas modificaciones en la organización de la producción, a la flexibilidad laboral al límite, y al aumento del desempleo y del empleo precario e informal.

Teniendo en cuenta que la primera noción de informalidad surge en los años setenta, para explicar el autoempleo en países africanos; y que desde esa década diversos enfoques teóricos intentaron explicar en países de América Latina y el Caribe la existencia, tanto de trabajadores por cuenta propia como de pequeñas unidades de producción, fuera de la lógica de la acumulación bajo el concepto de sector informal, en una época en la que las teorías del desarrollo se basaban en el dualismo entre un sector tradicional rural y uno moderno industrial que absorbería la mano de obra paulatinamente ${ }^{2}$, cabría preguntarse ¿cómo se define la informalidad del empleo en la etapa actual caracterizada por el desempleo, la flexibilidad y la precariedad laboral?, ¿es operativo el concepto de empleo informal para identificar y agrupar características específicas de ocupación?, ¿el concepto y criterios de medición del empleo informal pueden ser aplicables a países desarrollados y en desarrollo a pesar de las diferencias en la composición histórica de sus mercados de trabajo y de sus instituciones?

Para responder estas preguntas, el escrito expone, en primer lugar, la distinción conceptual entre economía subterránea y sector informal. La primera utilizada sobre todo en países europeos para distinguir actividades no declaradas, y la segunda, en países de África y América Latina para identificar principalmente al trabajo independiente. Luego, describe el origen del concepto de informalidad y los principales enfoques sobre el sector informal que dieron visibilidad a todas aquellas actividades económicas en pequeña escala, con escasa o ninguna división entre el trabajo y el capital como factores de producción, desarrolladas para la subsistencia, y en ocasiones como parte de la subcontratación que requería el proceso de acumulación. Un tercer apartado expone la evolución teórica y estadística del empleo informal según la OIT, institución que ha trabajado en su definición y medición hasta llegar al pleno esclarecimiento de su existencia en los asalariados. Debido a que la OIT no define criterios claros para identificar este empleo asalariado informal, en la última parte del escrito se proponen tres categorías para ello y dos rutas de medición de la informalidad.

Se concluye que existen suficientes elementos para distinguir el empleo informal y aplicar el concepto en economías con diferentes formaciones económicas y

2 Se pueden mencionar las teorías de Rostow (1967 y 1973) y de Lewis (1960 y 1971). Para Rostow, "el desarrollo es un proceso que discurre entre la sociedad tradicional y la sociedad de masas, siendo éstas las dos categorías polares". Por otra parte, Lewis plantea un modelo de dos sectores: uno capitalista y uno de subsistencia. Conforme se reinvierta el capital, el sector capitalista logrará absorber la mano de obra excedente en su totalidad. Para un análisis del dualismo en la teoría económica (De León, 1996, pp. 54-78). 
sociales, conformadas por empleo asalariado y no asalariado, lo que puede ayudar a visualizar las tendencias generales en las condiciones de ocupación.

\section{Economía subterránea y sector informal}

Al igual que ocurre con la noción de sector informal, no existe una sola definición de economía subterránea. No obstante, la mayor parte de los investigadores entiende que se refiere a actividades ilegales que no son registradas en la contabilidad nacional, por lo que la preocupación central ha sido crear los instrumentos que permitan un registro del PIB generado por estas actividades. De acuerdo con el Sistema de Cuentas Nacionales de Naciones Unidas de 1993, la economía subterránea se define, como:

Algunas actividades pueden ser a la vez productivas desde el punto de vista económico y totalmente legales (a condición de que cumplan con algunas normas o normativas), pero deliberadamente ocultadas a las autoridades públicas por distintos tipos de razones: a) evitar el pago del impuesto a la renta, del impuesto sobre el valor agregado o de otros impuestos; b) evitar el pago de las cotizaciones del seguro social; c) no tener que cumplir con algunas normas legales: salario mínimo, horario máximo, normas en materia de seguridad o de salud; d) evadir algunos procedimientos administrativos, tales como el hecho de llenar cuestionarios estadísticos u otros formularios administrativos. «Los productores que ejercen esta clase de actividades forman parte de lo que se llama 'la economía subterránea' (OCDE, 2007, p. 45).

Por otro lado, de acuerdo con la Resolución de la $15^{\mathrm{a}}$ Conferencia Internacional de Estadísticos del Trabajo de la OIT, 1993, el sector informal se define como:

Un conjunto de unidades que producen bienes o servicios principalmente con la finalidad de crear empleos e ingresos para las personas interesadas. Estas unidades tienen un bajo nivel de organización y operan a pequeña escala y de manera específica, con poca o ninguna división entre el trabajo y el capital como factores de producción. Las relaciones de empleo -cuando existen- se basan sobre todo en el empleo ocasional, los parentescos o las relaciones personales y sociales, más que en acuerdos contractuales que implican garantías en buena y debida forma (OIT, 2003, p. 2).

El denominador común que se ha encontrado en ambas categorías es la existencia de actividades no captadas por los registros fiscales. En las economías industrializadas donde impera el empleo regulado y un Estado estricto en su cumplimiento, la economía subterránea se identifica con actividades que practican la evasión fiscal y con actividades ilegales como el tráfico de drogas y la prostitución. La informalidad se ha entendido solo desde un punto de vista estadístico (economía no registrada, economía no observada, economía no oficial, economía no declarada, economía invisible) o delictivo (economía disimulada, economía sumergida, economía ilegal, 
economía clandestina, economía gris, economía escondida, economía subterránea, economía oculta, economía negra, economía irregular) (Francois Roubaud, 1995).

Por otro lado, en los países en desarrollo, el concepto de informalidad ha cobijado las características de un sector de la economía que está fuera del proceso de industrialización, cuya finalidad es la supervivencia y no la acumulación. Se trata del sector informal, que por sus características productivas y de relaciones de empleo no cumple con las normas legales entre las cuales está el pago de impuestos.

El Sistema de Cuentas Nacionales considera que el sector informal forma parte del sector de los hogares, distinguiéndose como "empresas de hogares" que no están constituidas en sociedad y por lo tanto no están registradas, pero reconoce que

Las actividades realizadas por las unidades de producción del sector informal no se realizan con la intención deliberada de eludir el pago de impuestos o de contribuciones a la seguridad social, o de infringir la legislación laboral y otras disposiciones legales o ciertas normas y procedimientos administrativos, por lo que el concepto de actividades del sector informal debería diferenciarse del de actividades ocultas o del de economía subterránea ( $15^{\text {a }}$ CIET, p. 2).

En última instancia, el límite conceptual entre ambos es muy difuso, entendiendo que el sector informal engloba actividades extralegales (fuera de la legalidad), y la economía subterránea actividades que no están permitidas por la ley. No obstante, existe una diferencia sustancial que atinadamente expresa Contreras Carbajal:

La diferencia estriba en que las actividades realizadas por los informales van más allá de la legalidad y su incumplimiento, obedecen a un comportamiento no consciente, producto de su quehacer económico, básicamente de supervivencia; mientras que en la economía subterránea es manifiesta la inclinación consciente a violentar el orden legal $(2015$, p. 72$)$.

En el estudio de la informalidad con base en estos conceptos Contreras Carbajal apunta a no olvidar que ambos fueron creados para corregir las insuficiencias que presentaba el Sistema de Contabilidad Social de las Naciones Unidas (el de economía subterránea fue acuñado por el Sistema de Cuentas Nacionales de Naciones Unidas y el de sector informal por la OIT) y, en ese sentido, era menester agrupar las actividades, definirlas y encontrar en su condición legal el denominador común para que fuesen operativos en términos estadísticos. No obstante, este conjunto de actividades representa diferentes problemas socioeconómicos, por lo que reducir su análisis a estas dos categorías privaría de encontrar caminos para su comprensión. Existe entonces la necesidad de "buscar otro tipo de elementos teóricos que posibiliten una comprensión más profunda de estos fenómenos" (Contreras, 2015, p. 95).

En esa tarea, y teniendo como interés principal el estudio de las condiciones de ocupación y no la condición legal de las actividades, el elemento teórico que se propone utilizar es el de empleo informal para identificar y esti- 
mar, en términos más precisos, algunos aspectos de la precariedad laboral ${ }^{3}$ que están presentes en el sector formal e informal, y que pueden identificarse en países desarrollados y no solo en actividades económicas de América Latina. En este sentido, se reconoce que los conceptos del sector informal y economía sumergida han surgido para comprender actividades y empleos tan distintos como lo son los niveles de desarrollo de los países donde se han utilizado. La creación y el uso de estos términos no tiene lugar solo con fines contables, sino que reflejan necesariamente un contexto económico y social. Así, el sector informal es un rasgo distintivo del subdesarrollo y encierra no solo condiciones de empleo y de productividad de las empresas, también refleja la estructura de acumulación y el funcionamiento de las instituciones.

La antesala del empleo informal como instrumento teórico y estadístico son los estudios sobre el sector informal que surgieron en la década de los setenta, y que comprendían este sector desde los siguientes ámbitos: a. la composición de las unidades productivas (en términos de tamaño, de productividad y de relaciones entre trabajadores y empresarios); b. los vínculos existentes entre el sector informal con el formal (la creación de unidades informales por parte de las grandes empresas para minimizar costos y evadir impuestos); y c. la condición legal de las actividades (su falta de registro como actividades formales y la no declaración de impuestos). Estos ámbitos son desarrollados por los enfoques teóricos que se exponen a continuación.

\section{Origen del concepto de informalidad y principales enfoques teóricos sobre el sector informal}

En la década de los setenta las teorías del desarrollo basadas en el dualismo entre un sector tradicional rural y uno moderno industrial comenzaban a ser insuficientes para explicar los nuevos fenómenos en el ámbito de la ocupación de los países en desarrollo.

El autoempleo aumentaba como consecuencia de la migración rural-urbana y del crecimiento demográfico, sin que el sector moderno pudiera absorber la mano de obra que demandaba empleo. Ante este panorama, la OIT planteó la necesidad de generar puestos de trabajo a través del Programa Mundial de Empleo (PME), y fue en el informe sobre las tareas realizadas con este objetivo en Kenya que, en 1972, surge el término "sector informal", inspirado a su vez en un trabajo del investigador Keit Hart sobre Ghana en 1970 (OIT, 1972; Hart, 1970).

Hart había propuesto un modelo dualista de oportunidades de ingreso de la fuerza laboral urbana, asociando lo formal con el empleo asalariado y lo informal con el empleo por cuenta propia. El informe sobre Kenya explicó la situación ocupacional

3 El concepto de precariedad laboral es más amplio (OIT, 2012a; Rodgers y Rodgers (1989) y Standing (1999 y 2011). 
observando también una dualidad entre un sector formal y uno informal, identificando al sector informal con aquellos trabajadores que se encuentran fuera del mercado laboral, desarrollan actividades de baja productividad y perciben un ingreso insuficiente.

Con esta primera visión sobre la informalidad, la OIT continuó haciendo diversos estudios con el objetivo de definir metodologías apropiadas para estimar su tamaño en los países en desarrollo y mejorar la situación ocupacional en las actividades informales. Durante la década de los ochenta, los estudios para América Latina se hicieron a través del Programa Regional de Empleo para América Latina y el Caribe (PREALC).

En la década de los noventa, la ONU, a través de las reuniones de la OIT y de la División de Estadística, formaliza el reconocimiento del sector informal y del empleo informal dentro de este, al constituir por primera vez una norma internacional para generar estadísticas del empleo en el sector y abrir los espacios para la integración de dichas estadísticas en los sistemas de contabilidad nacional. Gracias a esto, la medición del sector informal mejoró y puede incorporarse cada vez más en las economías de los países (INEGI, 2004).

Desde su origen, el concepto de sector informal ha dado lugar al análisis de las "nuevas" formas de producción y de empleo. Si bien, en principio ayudó básicamente a distinguir el empleo fuera de la relación salarial con la distinción de trabajadores por cuenta propia y la existencia de unidades económicas con poco capital y niveles de productividad, es a partir de su definición que se ha llegado a la conceptualización del empleo informal, entendido como la informalidad en el puesto de trabajo, independientemente del sector en el que tenga lugar, formal o informal, facilitando así su estudio en las economías desarrolladas distinguidas por la predominancia del empleo asalariado y de las grandes empresas.

Básicamente, se distinguen tres enfoques teóricos que, al describir el sector informal, establecen las actividades productivas y el empleo informales en diferentes ámbitos y con distintos “propósitos” en las economías (Contreras, 2016, p. 56).

\section{La perspectiva estructuralista}

La perspectiva estructuralista surge a mediados de la década de los setenta con el Programa Regional del Empleo para América Latina y el Caribe (PREALC) de la OIT, que emprendió estudios sobre la ocupación, exponiendo la dinámica de un sector informal que tenía origen en la oferta ilimitada de mano de obra en un contexto de modernización capitalista basado en la industrialización sustitutiva de importaciones.

Esta visión tenía como antecedente el pensamiento dualista entre un sector moderno y uno secundario que dominaba en esos años y que tenía sus orígenes en los 
trabajos de Lewis (1954) y de Harris y Todaro (1970). Se basaba en la dualidad del mercado de trabajo en el área urbana entre un sector formal y uno informal que componía el segmento inferior, surgía del proceso de transformación de las economías en desarrollo, y sería absorbido por el sector moderno cuando se llegara a los niveles de crecimiento esperados. Al no ocurrir tal expectativa, el PREALC reconoció al sector informal como una alternativa de empleo caracterizada por:

- Su facilidad de entrada a los mercados, dado el poco capital y la poca organización que requerían sus actividades.

- Su baja productividad y su lógica de subsistencia ${ }^{4}$. La ocupación de mano de obra abundante en estas actividades no permitía niveles de productividad capaces de generar un excedente que posibilitara la mejora en tecnología y en la acumulación, por lo que la lógica del sector informal, a diferencia del sector capitalista, era la de subsistencia.

De acuerdo con su concepción, propuso una composición del sector para estimarlo, básicamente calculando la población económicamente activa (PEA) ocupada en las categorías de:

- Autoempleados (excluyendo administradores y profesionales).

- Trabajadores familiares no remunerados y

- Trabajadores en pequeñas empresas que tienen hasta cinco empleados.

El dualismo observado por el PREALC se sustentaba en la distinción de dos sectores dentro del ámbito urbano, separados por las grandes diferencias en la organización para la producción y la lógica económica. Distinguía un sector urbano moderno (SUM), constituido por actividades productivas que disponen de las tecnologías más evolucionadas y por tanto mantiene unos niveles de productividad similares a los de los países desarrollados. Aunque concierne a actividades de industria y servicios, el subsector manufacturero ocupa un lugar central. Se consideran dentro de este sector los segmentos de población empleados en estas actividades que reciben los ingresos más elevados dentro de la población ocupada; y un sector informal urbano (SIU), que reúne actividades que emplean tecnologías simples o rudimentarias y que, por ello, alcanzan bajas productividades. Está conformado por una amplia gama de unidades productivas que van desde el pequeño taller hasta el puesto de venta ambulante. Agrupa entonces, por un lado, a trabajadores que reciben bien un salario, bien una remuneración en especie y que actúan bajo modalidades de contratación informal (no legal); por otro, a aquellos que se autoemplean en actividades de servicios de poca cualificación. La división entre capital y trabajo no es nítida, y por tanto la estratificación en su interior no obedece a la configuración de clases prevaleciente en el sector moderno (De León, 1996, pp. 93-94).

$4 \quad$ No se trata de un modelo de subsistencia directa sin actividades mercantiles, sino de actividades económicas sin fines de acumulación. 
La autora Clara Jusidman distingue el enfoque PREALC como un "enfoque de racionalidad productiva" que define al sector informal como

La suma de actividades caracterizadas por una lógica de producción propia y distinta de la vigente en la parte visible de la economía. Su racionalidad económica consiste en garantizar la subsistencia del grupo familiar, que difiere de la vigente en el sector formal, cuya motivación esencial es la acumulación ${ }^{5}$ (Jusidman, 1997).

Si bien la dualidad estaba implícita no entre lo rural y lo urbano, sino en las diferencias de organización productiva en un sector urbano moderno y uno urbano informal, Alejandro Portes, crítico de este enfoque y ponente de la visión neomarxista, observa en el planteamiento una expectativa de absorción del exceso de mano de obra de las zonas rurales por parte del SIU y a su vez, una absorción del SIU por parte del SUM. Es por eso que hace una crítica a la dualidad implícita y afirma que el modelo elaborado por el PREALC simplemente rebautizó la diferencia entre los sectores moderno y tradicional, formulada en su momento por Lewis:

Las recomendaciones del PREALC son, en esencia, tan simples como la imagen dualista de las economías periféricas que propone: el sector informal es un regazo del pasado; su completa absorción dentro de la economía formal requiere de inversiones sostenidas en actividades productivas durante largos periodos [...] El crecimiento sostenido de esta última debe absorber la mano de obra confinada a las actividades informales, conduciendo a su desaparición (Portes, 1995, p. 149).

Al observar la experiencia de industrialización y de tasas sostenidas de crecimiento en América Latina entre los años de 1950 y 1980, el autor neomarxista critica la creencia de que el sector moderno terminaría por absorber la mano de obra excedente, pues en este periodo no se redujo la PEA ocupada en el sector informal. Por el contrario, reconoce que, incluso previo a la década de los ochenta, la disminución de la demanda de mano de obra en el sector moderno comenzaba a ser clara, dando paso a la informalidad como alternativa de empleo, incluso para la población ocupada en el sector formal.

Al respecto, Vega y Kruijt afirman lo siguiente:

Puede distinguirse que la noción de informalidad ha despertado al menos dos expectativas. La primera, correspondiente a los años setenta, tendió a identificar al sector informal como un proceso en transición, constituido principalmente por migrantes rurales pobres, jóvenes y mujeres, que llegaban a ese sector en espera de incorporarse a las actividades formales. La segunda está mediada por la crisis económica de los años ochenta. Se desvanece la

5 Para que una actividad tenga lógica de acumulación, requiere transformar el dinero en capital una y otra vez, adquiriendo tanto fuerza de trabajo como medios de producción. De otra manera, se estaría hablando de una actividad en la cual el dinero solo se aprovecha como fondo de consumo, gastándose con la misma periodicidad con la que se obtiene. Esta es la reproducción simple para Marx, en su obra El capital, cap. XX. 
expectativa de la transición hacia la formalidad y en su lugar aparece la idea de que es también una transición de la formalidad hacia la informalidad, debido a las privatizaciones, el retiro de subsidios, la apertura de mercados $\mathrm{y}$, en general, al ajuste y a las políticas neoliberales que engendran nuevos pobres (2000, p. 197).

Los neomarxistas percibían que este enfoque estructuralista acentuaba el dualismo y no analizaba las interrelaciones entre las actividades formales e informales en un contexto de economía unificada, pero en realidad, el PREALC reconocía la subordinación que se establece a partir de una relación de complementariedad entre un sector formal y uno informal, parecida a la que se da en el modelo centro-periferia de inspiración cepalina, donde el sector informal "importaría" capital e insumos del sector formal, y "exportaría" bienes y servicios al sector formal ${ }^{6}$. En este sentido, distingue una "articulación subordinada" del SIU, determinada por el proceso de modernización capitalista que concentra el dinamismo de la economía en el sector moderno. A partir de esta relación, las posibilidades de crecimiento del sector informal dependerían del desarrollo del sector formal y su funcionalidad indirecta para el capital global sería la de posibilitar el acceso a ciertos medios de consumo a los trabajadores asalariados.

Dadas las similitudes entre el polo marginal de la economía y el sector informal, el reconocimiento de que no es secundario sino complementario es primordial. Si bien el polo marginal de la economía es similar al sector informal, ya que normalmente se identifica con poca acumulación y niveles bajos de ingreso, un aspecto que los diferencia es que el primero sirve a un mercado conformado por la propia población incorporada a ese nivel sin tener relación con el sector no marginal de la economía, mientras que el sector informal establece relaciones de intercambio también con el sector formal respecto al origen y destino de los bienes y servicios que produce y distribuye (Klein y Tokman, 1979). Las actividades informales desempeñan un papel importante en el proceso de distribución, en tanto que es a través del comercio y los servicios informales en pequeña escala que se llega a un porcentaje muy significativo de consumidores que no son exclusivamente los de menores ingresos. Además, demanda insumos del sector formal para la producción, sin que necesariamente se trate de la subcontratación por parte de empresas modernas. Estas actividades se encuentran, por consiguiente, integradas de manera estrecha al resto de la economía.

La relación de complementariedad no implica que ambos sectores se expandan o se reduzcan en un periodo determinado, ya que las posibilidades de crecimiento del sector informal no dependen únicamente del desarrollo del sector formal y del consumo de sus asalariados, sino también del intercambio entre la misma población del sector informal (Tokman, 1991).

6 Los términos importación y exportación se refieren a relaciones intersectoriales, es decir, a las compras y ventas que realiza el sector informal con el resto de la economía. 
Otras críticas a la visión PREALC por parte de los neomarxistas son:

- Que no se incluye en la medición a los trabajadores asalariados que están en el sector, lo cual lleva a no distinguir el empleo no protegido, ya que serían los asalariados los que estarían en condiciones de tener protección laboral.

- Que no se considera la posibilidad de estar empleado de manera simultánea en el sector informal y en el formal.

- Critican la llamada "facilidad de entrada", la cual estaría determinada por las bajas exigencias de capital y de cualificación. Plantean que diversos estudios han señalado que sí existen barreras para entrar al sector informal, tales como la existencia de lazos particularistas con la clientela, localización espacial de las actividades, acceso a fuentes no institucionales de crédito y la necesidad de permisos oficiales?

- Cuestionan el bajo nivel de ingresos en el sector. Nombran la existencia de estudios que demuestran que el capital invertido en las empresas informales ha excedido en ocasiones el de las empresas llamadas formales y que los trabajadores informales perciben mayores ingresos (Portes, 1995).

- Observan el criterio del tamaño de la empresa (unipersonal en el caso de los autoempleados o microempresas de hasta cinco personas), argumentando que este juicio es arbitrario e insuficiente, debido a que el autoempleo también existe en el sector formal.

Pese a todas estas observaciones, el enfoque PREALC proporciona la base para el análisis del empleo informal al definir categorías y condiciones de ocupación.

\section{La perspectiva neomarxista}

Esta perspectiva se encuadra dentro del marxismo debido a que entiende que el sistema capitalista promueve la existencia de mano de obra excedente, constituida por el sector informal para abaratar costes de producción y posibilitar la acumulación. Sostiene que está íntimamente relacionado con el sector moderno de la economía a través de la subcontratación no regulada. De esta manera, el sector informal sería la forma ilegal en que se utiliza el trabajo en el sistema capitalista. Definen al sector informal como "todas las actividades con ingresos-ganancias no reguladas por el Estado, en contextos donde actividades similares sí lo están” (Portes, 1995, p. 131).

Este razonamiento se ajusta más a economías donde la división del trabajo ha alcanzado un nivel significativo, dando lugar a pequeñas empresas con trabajadores fuera de la regulación, que a economías atrasadas en las que abunda el autoempleo en la venta de productos y servicios, y que juega también un papel en la acumulación desde el ámbito de la realización y no de la producción de valor.

$7 \quad$ Standing (1974), Hart (1973), Peattle (1976); citados en Tokman y Klein, 1979, cap. I. 
En este sentido, Tokman expresa que en los países de América Latina las interrelaciones del sector informal con el resto de la economía se ponen de manifiesto por medio de la compra de bienes finales para su distribución minorista posterior, siendo los comerciantes ambulantes los que en mayor medida compran la mercancía directamente a la fábrica o al mayorista en forma de stocks (Tokman, 1991, p. 99).

Dada la dificultad de contar con datos sobre la regulación de los empleados, la corriente neomarxista asume que todos los trabajadores de pequeñas empresas presentan rasgos fuera de la legalidad laboral (protección contra despidos arbitrarios, compensación por desempleo, seguro por accidentes, ausencias pagadas, pensiones por jubilación) y opta por medir el sector informal con base en la proporción de la PEA urbana que trabaja en estas empresas.

Ya que consideran que el sector informal está íntegramente ligado al sector moderno como parte de una misma economía, afirman que en periodos de expansión económica y asumiendo niveles constantes de regulación estatal, se esperaría que se desarrollen ambos sectores, mientras que en periodos de contracción los dos sufrirán.

Lo que hay que reconocer de este enfoque es su clara distinción de una articulación entre el sector moderno y el sector informal en un contexto global de acumulación capitalista que nos induce a comprender al SIU bajo un contexto de concentración y centralización del capital, donde las multinacionales son las que finalmente determinan los precios y los mercados. Tokman expresa que

...las relaciones entre los sectores formal e informal deberían ser analizadas como parte de un sistema subordinado de interrelaciones a nivel internacional. El excedente económico generado en su interior es transferido al resto de la economía como paso intermedio del traslado de excedentes desde la periferia a los países centrales (1991, p. 93).

Por último, destaca de esta corriente, por un lado, su visión histórica de la informalidad urbana, ya que no la plantean como reciente sino como una expresión inicial del desarrollo del capitalismo y, por otro, el papel fundamental que da al Estado como responsable de la institucionalización de leyes laborales que originan un sector informal y lo separan de uno regulado. Portes afirma al respecto que, ciertamente, la articulación de diferentes formas de producir, reflejada en la división formal/informal es, en último análisis, un proceso político dependiente del control estatal, por lo que no pueden analizarse en términos exclusivamente económicos. Su existencia y funcionamiento dependen de las prácticas estatales institucionalizadas que reflejan la historia de la lucha de clases. A través de estas acciones se puede establecer y hacer cumplir las leyes laborales o abandonarlas. Afirma incluso que, si el sector informal existe, es porque el Estado ha institucionalizado los resultados de la lucha de clases a través de las leyes laborales y de mecanismos para su ejecución (Portes, 1995, pp. 40-43). El Estado debería garantizar entonces la seguridad laboral en ambos sectores, cerrando con ello, según su concepción, la división entre lo formal y lo informal. 


\section{El enfoque neoclásico}

Este enfoque afirma que el sector informal es producto de las políticas intervencionistas del Estado. La excesiva regulación que este impone a los empresarios para desempeñar sus actividades lleva a optar por trabajar fuera de la legalidad, originando e incentivando al sector. Hernando De Soto, economista peruano, es el principal autor de esta perspectiva. Con sus estudios sobre el sector informal a finales de la década de los ochenta en Lima, Perú, lo identificó con todas aquellas actividades económicas extralegales capaces de mantenerse a pesar de la presencia de un Estado aplastante. Este autor estimó la actividad informal con base en un levantamiento censal de los trabajadores informales en las áreas de vivienda, transporte y pequeño comercio. Rossini y Thomas señalan que sus estimaciones no son válidas debido a un uso inadecuado de medidas monetarias en las transacciones del Producto Nacional Bruto (PNB), diseñadas para ser utilizadas en economías desarrolladas, así como de modelos poco especificados y métodos econométricos antiguos (1988; Portes, 1995, p.130).

Otra crítica que se hace por parte de los neomarxistas es que la estimación no incluye a las actividades manufactureras de subcontratación ni distingue entre trabajadores y empresarios informales.

Esta perspectiva expone con más contundencia la dualidad entre un sector legal y uno no regulado; no obstante, carece de un análisis de la composición interna del sector informal y de las relaciones entre los sectores formal e informal y su visión es reduccionista al considerar que el sector informal solo se origina y se rige por la presencia de la excesiva regulación exigida por el Estado.

Por otra parte, no es del todo cierto que las actividades informales se encuentren totalmente fuera de la regulación. De hecho, muchas de ellas necesariamente deben registrar de inicio su actividad para poder desempeñarla e incluso para acceder a beneficios, tales como el crédito. De Soto reconoce que las actividades informales solo pueden acceder a los recursos y los mercados más dinámicos a través de la maquinaria legal e institucional vigente (1986). Por ello, en la medida en que una actividad informal crezca y no le sea conveniente estar fuera de la reglamentación, avanzará hacia su cumplimiento.

Para identificar a las actividades como legales, habría que definir qué es lo legal y hasta qué punto son viables los procedimientos legales en cada actividad del sector informal. Víctor E. Tokman distingue tres aspectos de la legalidad:

- El primero se refiere al reconocimiento como actividad comercial, lo que generalmente obliga al registro $\mathrm{y}$, en algunos casos a inspecciones sanitarias y de seguridad.

- El segundo se relaciona con los impuestos e implica registrarse para ser identificado como contribuyente potencial, pero también genera un compromiso permanente de pagar los diferentes impuestos según cada legislación nacional. 
- El tercer aspecto se refiere a los asuntos laborales y abarca, desde registrarse como empleador y celebrar contratos con los empleados hasta asegurar beneficios no salariales como por ejemplo vacaciones anuales, horarios de trabajo y aportes al seguro social.

Al respecto, el PREALC afirma que el sector informal opera entre lo subterráneo y lo legal y, de esta forma, el productor informal tiene acceso a las reglamentaciones que considera importantes, al mismo tiempo que minimiza los riesgos de la ilegalidad. Por lo tanto, operar más allá de la reglamentación, que es una característica común atribuida a la informalidad por todas las interpretaciones, constituye un asunto relativo (Tokman, 2001). Además, no solo las actividades informales están fuera de la reglamentación, sino también las "formales", aunque sea de manera parcial. Tokman sostiene que lo que prevalece son las llamadas "zonas grises", es decir, zonas intermedias entre la legalidad y la ilegalidad (Tokman y Klein, 1996). Por lo anterior, la definición y comprensión del sector informal no puede reducirse a su supuesta condición legal.

Sin duda, una reglamentación (incluso en el sector formal de la economía) sería positiva para la sociedad en su conjunto, pero no puede definir por sí misma al sector informal. En todo caso, esta visión neoliberal ha sido la más difundida y la que se ha adoptado para la comprensión de toda actividad "fuera" del sector moderno y de las normas administrativas de las economías.

\section{Evolución teórica y estadística del empleo informal según la Organización Internacional del Trabajo (OIT)}

La OIT ha seguido las recomendaciones del Sistema de Cuentas Nacionales (SCN) de $1993^{8}$ para trabajar en el estudio, la medición y la inclusión del empleo informal como concepto estadístico internacional. En este sentido, las Conferencias Internacionales de Estadísticos del Trabajo (en lo sucesivo CIET) han trabajado en la definición y medición del empleo informal hasta llegar al pleno esclarecimiento de su existencia en el sector formal de la economía, lo que ha abierto la posibilidad de estudiar aspectos informales del empleo a partir de registros estadísticos oficiales en países desarrollados.

Los acuerdos más importantes que se observan en el cronograma de la informalidad como concepto estadístico de la OIT, son los siguientes:

- La definición de sector informal, que se acordó en la $15^{\mathrm{a}}$ CIET de 1993 y que se ha descrito anteriormente.

8 El Sistema de Cuentas Nacionales fue elaborado por la Comisión de las Comunidades Europeas-Eurostat, el FMI, la OCDE, las Naciones Unidas y el Banco Mundial, y en él se inserta plenamente la Unión Europea a través del Sistema Europeo de Cuentas (SEC-1995). 
- La distinción de las empresas que componen el sector informal y cuyos empleos son informales. Existía, por un lado, la visión de que la informalidad de las empresas está necesariamente acompañada de la falta de registro y, por otro, la posición de que la falta de registro puede ser solo una característica, pero no un criterio único de definición considerando que en la práctica puede haber unidades del sector informal que están registradas, por lo que el principal criterio debería ser el tamaño de las empresas de acuerdo con el número de ocupados ${ }^{9}$. La resolución de la $15^{\mathrm{a}} \mathrm{CIET}$ incorporó ambos puntos de vista como criterios para distinguir las empresas del sector informal: la ausencia de registro y el número de personas ocupadas. No obstante, no especificó el límite de tamaño para las empresas informales, de modo que puede variar en función de las necesidades de los países e incluso de una rama de actividad económica a la otra dentro de un mismo país ${ }^{10}$. En cuanto al término "empresa" en la definición incluía tanto a unidades que contratan a trabajadores (empleadores) como a unidades administradas por particulares que son trabajadores independientes (trabajadores por cuenta propia) (OIT, 2013, p. 18). La $15^{\text {a }}$ CIET expresa que el tipo de datos que hay que recopilar sobre el sector informal depende en gran medida de las circunstancias específicas de cada país, de los métodos utilizados, del uso que vaya a darse a las estadísticas, y de la viabilidad práctica de la recolección de datos.

- La Clasificación Internacional de la Situación en el Empleo que también se acordó en la $15^{\text {a }}$ CIET (en lo sucesivo CISE-93). Clasifica las situaciones de empleo en las que se encuentran los trabajadores dentro del mundo del trabajo. Pueden ser empleos asalariados y empleos independientes o no asalariados, conformados por las siguientes categorías:

\section{Asalariados}

- Asalariados regulares con contratos sin límites de tiempo: son «asalariados regulares» que tienen contratos que solo se pueden terminar por causas determinadas, tales como la incompetencia, la mala conducta o por razones económicas de acuerdo con la legislación o costumbres nacionales.

- Trabajadores ocasionales: son los trabajadores que tienen un contrato de trabajo implícito o explícito que no se espera que continúe más allá de un periodo corto, cuya duración deberá determinarse de acuerdo con las circunstancias nacionales.

9 "...La lógica subyacente del criterio basado en el número de personas empleadas es que muchas veces las empresas con un número de empleados inferior a un umbral están exentas, en virtud de la legislación laboral y de seguridad social, del registro de empleados y probablemente no las abarca la recaudación de impuestos ni el control de la aplicación de la legislación laboral..." (OIT, 2012, p. 4).

10 Para una definición de las empresas del sector informal de acuerdo al país consultar La medición de la informalidad: Manual estadistico sobre el sector informal y el empleo informal. OIT, Ginebra 2013, p. 26. 
- Trabajadores estacionales: son los trabajadores que tienen contratos explícitos o implícitos de trabajo cuya duración y momento en que se efectúa dicho contrato están influenciados significativamente por factores estacionales, tales como el ciclo del clima, los días festivos y las cosechas agrícolas.

- Asalariados aprendices o en formación: de acuerdo con las circunstancias nacionales, los países pueden clasificar como asalariados aprendices o en formación a los trabajadores que tienen un contrato de «empleo asalariado» implícito o explícito que especifica que toda o una parte de su remuneración consiste en una capacitación para el ejercicio de un oficio o profesión.

\section{No asalariados}

- Empleadores: son aquellos trabajadores que, trabajando por su cuenta o con uno o más socios (los socios no son necesariamente miembros de la misma familia u hogar), tienen el tipo de empleo definido como «empleo independiente» y que, en virtud de su condición de tales, han contratado a una o a varias personas para que trabajen para ellos en su empresa como «asalariados» a lo largo de un periodo continuo que incluye el periodo de referencia.

- Trabajadores por cuenta propia: son aquellos trabajadores que, trabajando por su cuenta o con uno o más socios (los socios no son necesariamente miembros de la misma familia u hogar), tienen el tipo de empleo definido como «empleo independiente» y no han contratado a ningún «asalariado» de manera continua para que trabaje para ellos durante el periodo de referencia.

- Miembros de cooperativas de productores: son los trabajadores que tienen un «empleo independiente» en una cooperativa que produce bienes y servicios, en el que cada miembro participa en pie de igualdad con los demás miembros en la determinación de la organización de la producción, ventas u otras actividades del establecimiento, en las inversiones y en la distribución de los beneficios de este entre sus miembros.

- Trabajadores familiares auxiliares: son aquellos trabajadores que tienen un «empleo independiente» en un establecimiento con orientación de mercado, dirigido por una persona de su familia que vive en el mismo hogar, pero que no pueden considerarse como socios, debido a que el nivel de dedicación a la operación del establecimiento, en términos de tiempo de trabajo u otros factores que deben determinarse de acuerdo con circunstancias nacionales, no es comparable con aquel del jefe del establecimiento.

La definición de empleo informal como norma estadística internacional. En la $17^{\mathrm{a}}$ CIET del año 2003 se manifestó la importancia de reconocer el empleo informal en general, independientemente del sector donde tuviese lugar (formal o informal). Así, la definición de empleo informal comprende "el número total de empleos infor- 
males [...], ya se ocupen éstos en empresas del sector formal, empresas del sector informal, o en hogares, durante un periodo de referencia determinado" (17 ${ }^{\mathrm{a}}$ CIET, OIT, 2003, p. 2). Incluye la siguiente clasificación del empleo por tipo de unidad de producción (formal o informal):

\section{En el sector informal}

- Trabajadores por cuenta propia. Dueños de sus propias empresas del sector informal o que producen bienes exclusivamente para el propio uso final del hogar.

- Empleadores dueños de sus propias empresas.

- Trabajadores familiares auxiliares.

- Miembros de cooperativas de productores que no están formalmente constituidas en entidades legales.

- Asalariados que tienen un empleo informal.

\section{En el sector formal}

- Asalariados que tienen empleos informales en empresas del sector formal o como trabajadores domésticos asalariados empleados por hogares.

- Trabajadores familiares auxiliares.

- Trabajadores por cuenta propia que producen bienes exclusivamente para el propio uso final de su hogar.

El avance más importante de la informalidad como concepto estadístico es el pleno esclarecimiento de la existencia de asalariados con empleos informales en empresas del sector formal, pues esto permite extender su estudio a países donde impera el empleo asalariado y las empresas están constituidas en entidades legales. Sin embargo, la OIT no propone criterios específicos para su estimación.

\section{La estimación del empleo informal}

Los planteamientos de la OIT respecto a la estimación del empleo informal se sustentan en la Clasificación Internacional sobre la Situación en el Empleo (CISE1993), que recoge algunas categorías ya identificadas por las primeras teorías sobre el sector informal, como la de trabajador por cuenta propia y trabajador familiar auxiliar. La OIT considera que según las circunstancias nacionales y el uso que ha de darse a los datos, “....se podrá reunir información sobre diversas formas de empleo atípicas o precarias [...] A tal efecto todas las personas ocupadas, sea en el sector informal o fuera de él, deberían clasificarse en función de la situación en el empleo..." (CIET, 1993, p. 8). Así, una primera ruta que se propone para estimar el empleo in- 
formal es identificando las situaciones de empleo no remunerado, reconocidas por la OIT dentro del sector informal: empleadores, trabajadores por cuenta propia, miembros de cooperativas de productores, trabajadores familiares auxiliares.

Un gran número de países ha incluido estadísticas nacionales de acuerdo con esta clasificación, por lo que no es difícil encontrar elementos para estimar estas categorías a nivel nacional. La OIT recoge datos sobre el empleo informal considerando estas situaciones de empleo. El próximo cuadro representa la proporción del empleo informal en el empleo total del sector no agrícola. Países como Colombia, México y Paraguay tienen porcentajes de empleo informal muy elevados $(47,3 \%, 52,2 \%$ y $65,4 \%$, respectivamente), mientras que El Salvador, Turquía y la Federación Rusa ocupan a menos del $25 \%$ de la población económicamente activa, aunque la OIT advierte que las observaciones imputadas no se basan en datos nacionales, están sujetas a una gran incertidumbre y no deben utilizarse para comparaciones o clasificaciones de países.

Figura 1. Empleo informal en países seleccionados, años 2016 a 2018

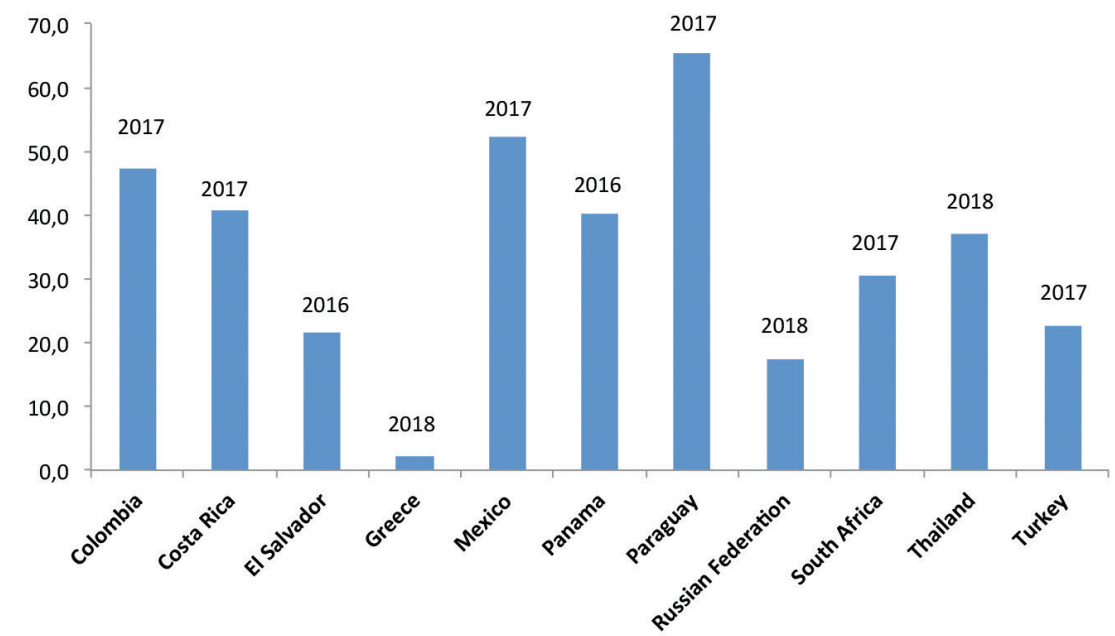

Fuente: Elaboración propia con datos de la OIT. Incluye trabajadores por cuenta propia, empleadores, miembros de cooperativas, trabajadores familiares auxiliares o empleados con empleos informales.

La segunda vía de medición que se propone es cuantificar a los asalariados con empleos informales en empresas del sector formal y a los trabajadores por cuenta propia registrados. Para cuantificar a los primeros deberían existir criterios definidos, pero no es así, aunque la siguiente definición de la OIT esboza ciertas características de ocupación:

Los asalariados tienen un empleo informal si su relación de trabajo, de derecho o de hecho, no está sujeta a la legislación laboral nacional, el impuesto sobre 
la renta, la protección social o determinadas prestaciones relacionadas con el empleo (preaviso al despido, indemnización por despido, vacaciones anuales pagadas o licencia pagada por enfermedad, etc.). Las razones pueden ser las siguientes: la no declaración de los empleos o de los asalariados; empleos ocasionales o empleos de limitada corta duración; empleos con un horario o un salario inferior a un límite especificado (por ejemplo para cotizar a la seguridad social); el empleador es una empresa no constituida en sociedad o una persona miembro de un hogar; el lugar de trabajo del asalariado se encuentra fuera de los locales de la empresa del empleador (por ejemplo, los trabajadores fuera del establecimiento y sin contratos de trabajo), o empleos a los cuales el reglamento laboral no se aplica, no se hace cumplir o no se hace respetar por otro motivo (OIT, 2003, p. 3).

Figura 2. Empleo no remunerado en países seleccionados 1991-2019

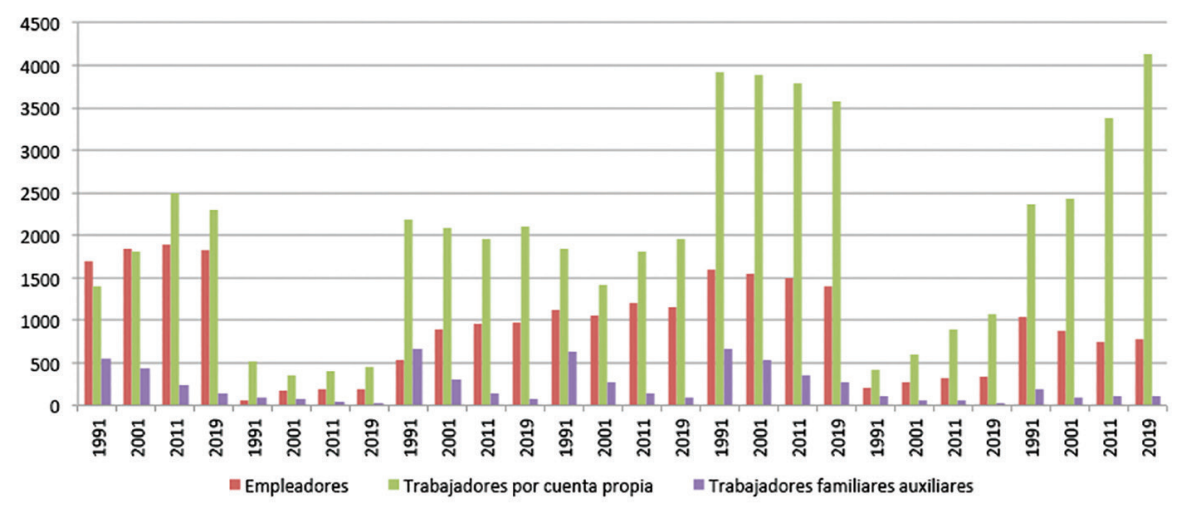

Fuente: Elaboración propia con datos de la OIT.

De acuerdo con esta definición, los asalariados tienen entonces un empleo formal cuando trabajan en actividades o empresas constituidas, que declaran su actividad, donde los empleos no son ocasionales, se establecen relaciones contractuales, se tiene un horario especificado y un salario suficiente para cotizar a la seguridad social, cuentan con un establecimiento, y cumplen con el reglamento laboral. Pero esta definición no considera que, en las empresas formales, el empleo ocasional también existe, normalmente bajo una relación contractual temporal, que el horario laboral puede reflejar el subempleo, y que el salario, si bien llega a niveles de cotización, puede ser menor al mínimo legalmente establecido. Además, suele no cumplirse con el reglamento laboral. Así, lo que existe, sobre todo en países desarrollados, es una informalidad legislada del empleo asalariado, al menos en lo que se refiere a contratos de duración determinada o temporales, empleo parcial involuntario, y bajos salarios. En este sentido, los tres criterios que se proponen para identificar la informalidad del empleo asalariado son los siguientes: 
Asalariados con contrato de duración determinada. Sea escrito o de palabra, la modalidad de contratación determina el grado de estabilidad laboral, la ausencia o insuficiencia de protección social y la inseguridad o vulnerabilidad económica de los trabajadores. Incluye la certidumbre sobre la continuidad en el trabajo; el control sobre el trabajo (determinación de salarios, condiciones laborales); y la protección legal (despidos injustos, prácticas laborales no aceptables, seguridad social) (OIT, 2013). Así, la informalidad del empleo de acuerdo con el contrato de trabajo puede incluir «trabajadores ocasionales», «trabajadores en formación» ${ }^{11} \mathrm{y}$ asalariados regulares ${ }^{12}$ cuyos contratos de trabajo permiten a la empresa terminar el acuerdo con corto plazo de aviso. En suma, se trata de trabajadores temporales cuya condición de vulnerabilidad en cuanto a estabilidad y derechos laborales, determinados por su modalidad de contratación, los ubica en un ámbito de empleo informal. Así lo expresa la OIT en uno de sus documentos de trabajo:

El tema de los trabajadores ocasionales es complejo, pues puede suponer un espacio de informalidad en medio de relaciones formales. En algunos casos esta ocasionalidad se admite de forma tan amplia que su duración sería equivalente a la de trabajadores temporales, en unos casos, o a la de trabajadores de temporada, en otros. En ambos casos, los trabajadores ocasionales pueden coexistir en la misma empresa con trabajadores fijos y formales, éstos con derechos plenos y aquellos sin más derecho que el salario y en el mejor de los casos, con alguna cobertura respecto del riesgo de accidente de trabajo (OIT, 2005, p. 41).

Asalariados subempleados. Es la informalidad determinada por la medición del tiempo de trabajo. De manera concreta, se trata del empleo a tiempo parcial, que de acuerdo con la OIT forma parte del subempleo por insuficiencia de horas de trabajo y se constituye por personas con un empleo que reúne los siguientes criterios ${ }^{13}$ :

a) Desear trabajar más horas. Aquí deben distinguirse aquellos que han buscado activamente trabajar horas adicionales de los que no lo hicieron.

b) Estar disponibles para trabajar más horas, es decir, poder efectivamente hacerlo durante un período posterior especificado, en función de las oportunidades de trabajo adicional que se presenten.

11 Recordemos que, según la CIET-93, los trabajadores ocasionales son los trabajadores que tienen un contrato de trabajo implícito o explícito que no se espera que continúe más allá de un periodo corto, cuya duración deberá determinarse de acuerdo con las circunstancias nacionales; y los asalariados en formación son los trabajadores que tienen un contrato de «empleo asalariado» implícito o explícito que especifica que toda o una parte de su remuneración consiste en una capacitación para el ejercicio de un oficio o profesión.

12 Los asalariados regulares según la CIET-93 son los que tienen contratos que solo se pueden terminar por causas, tales como la incompetencia, la mala conducta o por razones económicas de acuerdo con la legislación o costumbres nacionales.

13 En esta definición, el concepto de tiempo de trabajo es el de horas efectivamente trabajadas. De acuerdo con la Resolución sobre la medición del tiempo de trabajo de la $18^{\mathrm{a}} \mathrm{CIET}$, "las horas efectivamente trabajadas se refieren al tiempo dedicado al desempeño de actividades que contribuyen a la producción de bienes o servicios durante un período de referencia determinado, ya sea corto o largo. Las horas efectivamente trabajadas se aplican a todos los tipos de trabajos (dentro y fuera de la frontera de producción del SCN) y no están vinculadas con conceptos administrativos o jurídicos". Por otra parte, el valor umbral de horas se basa en la frontera establecida entre la ocupación a tiempo completo y a tiempo parcial, de acuerdo con la Resolución sobre las estadísticas del trabajo, la ocupación y la subutilización de la fuerza de trabajo (19 Conferencia Internacional de Estadísticos del Trabajo, OIT, 2013). 
c) Haber trabajado menos de un límite de horas determinado, es decir, las personas cuyo número de horas efectivamente trabajadas en todos los empleos durante el período de referencia era inferior a un límite definido en función, por ejemplo, de la frontera entre empleo a tiempo completo y empleo a tiempo parcial o al horario laboral establecido en la legislación correspondiente, convenios colectivos, acuerdos sobre jornada de trabajo o prácticas laborales nacionales (OIT, 1998).

Asalariados con ingresos menores al salario mínimo establecido. El nivel de ingresos ha sido un elemento de medición del empleo informal debido a que determina el trabajo decente en el mundo ${ }^{14}$. Uno de los principales indicadores de bajos ingresos son los trabajadores pobres, que se definen como "personas con empleo que viven en un hogar cuyos miembros están por debajo del umbral de la pobreza definido a escala nacional" (OIT, 2009, p. 23). El umbral de pobreza se define a su vez como "el nivel de ingresos mínimo que se considera necesario para tener una calidad de vida adecuada en un país determinado" (OIT, 2009, p. 24). Dicho nivel generalmente es el salario mínimo ${ }^{15}$, por lo que los asalariados informales serían aquellos que perciben ingresos menores al salario mínimo establecido en el país de que se trate.

La utilización de estos tres criterios de identificación del empleo asalariado informal puede ser especialmente útil para países desarrollados. Conceptualmente, hablar de trabajo informal puede resultar confuso en estas economías, debido a que su marco teórico ha sido el de economía subterránea o sumergida, pero, en términos operativos, una categorización del empleo asalariado informal puede ayudar a identificar y estimar aquellos trabajadores que, a pesar de estar en empresas formales, no dejan de ser vulnerables. Al respecto, la OIT expresa que "especialmente los países desarrollados podrían optar por limitar la medición del empleo informal a los empleos asalariados" (OIT, 2012, p. 11), y que "los criterios operativos para definir empleos asalariados informales deberían determinarse en función de las circunstancias nacionales y de la disponibilidad de información" (OIT, 2003, p. 15).

Por otra parte, en los trabajadores por cuenta propia, la OIT solo considera a aquellos que producen bienes exclusivamente para el propio uso final de su hogar; sin embargo, tanto en países desarrollados como en desarrollo, existen trabajadores independientes registrados y constituidos legalmente que producen para el mercado y

14 El conjunto de Indicadores de Trabajo Decente fue elaborado según las orientaciones recibidas en la reunión tripartita de expertos sobre la medición del trabajo decente, celebrada en Ginebra entre el 8 y el 10 de septiembre del 2008. Consultarlos en Guía sobre los nuevos indicadores de empleo de los objetivos de desarrollo del milenio, OIT, 2009.

15 En el informe de la $79^{\text {a }}$ CIET de 1992 se expresa que "el salario mínimo, bajo cualquier forma que sea calculado, por hora o por rendimiento, constituye una base que no puede ser disminuida y cuya aplicación está garantizada por la ley [...] El establecimiento de un sistema de salario mínimo frecuentemente es presentado como el medio para asegurar a los trabajadores (y en ciertos casos a sus familias) un mínimo vital que les permite subvenir a sus necesidades (y a las de sus familias), lo que a veces trae consigo la utilización del término "salario mínimo vital". La puesta en práctica de tal concepto implica una actitud, una política que tiende a mejorar la situación material de los trabajadores y garantizarles un mínimo vital o un nivel mínimo de vida adecuado, compatible con la dignidad humana o suficiente para cubrir las necesidades esenciales de los trabajadores (OIT, 1992, p. 13). 
que suelen tener ayuda de trabajadores familiares no remunerados, cuya presencia ya refleja la categoría restante de empleo informal. En España, por ejemplo, estos trabajadores están "protegidos" por el Estado en términos laborales al estar registrados en la Seguridad Social dentro del Régimen Especial de Trabajadores Autónomos, pero, en la práctica, sus beneficios son insuficientes durante y cuando cesa su actividad, y su registro incluye a los trabajadores familiares auxiliares sin desglosar datos específicos sobre las condiciones laborales de estos, lo que constituye una informalidad del empleo ${ }^{16}$. Así, la propuesta es incluir en el empleo informal a trabajadores independientes registrados que perciban menos del salario mínimo establecido y visualizar si en su registro está encubierta la ocupación de trabajadores familiares sin pago.

En cuanto a la estimación de los trabajadores domésticos asalariados, la OIT afirma que, debido a su carácter atípico, se considera un "grupo de difícil cobertura" por la seguridad social y, por tanto, de difícil cobertura estadística. Estima que del total de 67 millones de trabajadoras domésticas en el mundo, más de 60 millones están excluidas de la cobertura de la seguridad social. Es en los países asiáticos y latinoamericanos donde hay más trabajadores domésticos $(41 \%$ y $27 \%$, respectivamente), mientras que en los países desarrollados solo son un 10\% (OIT, 2016). En estos últimos, la inclusión del trabajo doméstico en las legislaciones nacionales es generalmente parcial y su sistema de protección social débill7. Así, la aplicación de medidas para mejorar su situación de empleo es urgente, tal y como lo propone la institución en el Convenio n ${ }^{\circ} 189$ (OIT, 2011).

\section{Conclusiones}

Las condiciones de empleo adquieren importancia en la medida en que crecen el desempleo y las alternativas de ocupación fuera de la relación salarial y la flexibilidad e informalización del trabajo para obedecer a una demanda en constante cambio en un contexto de crisis recurrentes del sistema económico.

La incertidumbre en este contexto se acompaña de falta de conceptos que definan las realidades en el ámbito del trabajo. Este escrito se ha propuesto contribuir al análisis teórico del empleo informal y a la definición de criterios para su estimación, teniendo como antecedente un uso del término informal tan múltiple y difuso que ha dado lugar a la defensa de su nula utilidad como categoría analítica (Contreras, 2015; Connolly, 1990) y, al mismo tiempo, a su aplicación continua para explicar distintas

16 Se entenderá como trabajador por cuenta propia o autónomo aquel que realiza de forma habitual, personal y directa una actividad económica a título lucrativo, sin sujeción por ella a contrato de trabajo y aunque utilice el servicio remunerado de otras personas, sea o no titular de empresa individual o familiar [...] Están incluidos los cónyuges y familiares hasta el tercer grado inclusive, por consanguinidad, afinidad y adopción que colaboren con el trabajador autónomo de forma personal, habitual y directa y no tengan la condición de asalariados (Ministerio de Empleo y Seguridad Social, Gobierno de España).

17 Véase como ejemplo el caso de España en "Las reformas de la regulación del trabajo doméstico por cuenta ajena en España”, de Elena Desdentado Daroca, Universidad Nacional de Educación a Distancia, 2015. 
condiciones de empleo y de unidades productivas en regímenes económicos de todo el mundo.

El análisis del empleo informal siempre ha llevado intrínseca la división entre un sector formal y uno informal en el ámbito económico, entre pequeñas o unipersonales actividades con poca o nula productividad y grandes sectores productivos, entre empresas legalmente constituidas y registradas y empresas sin registro, entre actividades legales y las que están fuera de la legalidad, entre empresas que declaran impuestos y empresas que no lo hacen, entre actividades económicas realizadas en la calle y las que se realizan bajo techo, entre países desarrollados y en desarrollo. Los múltiples términos en torno al concepto, construidos por la misma institución que le ha dado origen e impulsa su definición y su operatividad, la OIT, han hecho que se pierda (economía informal, sector informal, empresas del sector informal). Este trabajo esclarece la conformación del empleo informal y reafirma que puede existir en cualquier actividad económica dejando de lado estas dicotomías. Reafirma también que existe en el trabajo remunerado de los sectores más constituidos y propone categorías específicas para la estimación de este empleo asalariado informal, al mismo tiempo que propone retomar las situaciones de trabajo no remunerado como primer vía de estimación del empleo informal.

La primera conclusión que se deriva de las preguntas iniciales es que la informalidad en la etapa actual de desempleo, precarización y flexibilidad del trabajo se distingue por la existencia de empleo asalariado informal en los sectores económicos más dinámicos e inscritos en las legislaciones vigentes en términos públicos y de empleo de los países. La OIT reconoce a estos trabajadores como "asalariados que tienen empleos informales en empresas del sector informal" (Directriz número 5 (i) de la Decimoséptima CIET-OIT, 2003), pero no especifica criterios para medirlo, por lo que se concluye, además, con una propuesta de categorías para su estimación que hace operativo el concepto en el empleo asalariado y, por tanto, aplicable en países desarrollados donde este predomina. Asimismo, la propuesta de retomar las situaciones de trabajo no asalariado como vía de estimación hace operativo el término en toda economía donde este tenga lugar.

La extensión de la informalidad del empleo que en definitiva se distingue no desconoce los determinantes históricos de los mercados de trabajo de los países ni las diferencias en sus formaciones económicas, sociales e institucionales, solo esclarece que el empleo no remunerado, temporal, a tiempo parcial y de bajos ingresos puede existir en países con diferentes niveles de desarrollo y constituyen características importantes de la ocupación.

Por último, se considera que el intento de rescatar conceptos tan complejos en lugar de optar por su inutilidad o su omisión ayuda a valorar y a poner en práctica la ardua tarea de los estudiosos del mundo del trabajo. En este caso, de la OIT. 


\section{Referencias}

Connolly, P. (1990). Dos décadas de sector informal. Sociológica. Revista del Departamento de Sociología. 5(12), 1-15. Universidad Autónoma Metropolitana.

Contreras, J.J. (2015). Enfoque crítico sobre las teorías del sector informal urbano en América Latina. Universidad Autónoma Metropolitana. Editorial Itaca.

Contreras, T. (2016). El empleo informal del colectivo latinoamericano con más residentes en España ante la crisis (una aproximación a su medición, 2005-2014). (Tesis de doctorado). Universidad Complutense de Madrid, Madrid, España.

De León, O. (1996). Economía informal y desarrollo: Teorías y análisis del caso peruano. Instituto Universitario de Desarrollo y Cooperación.

Harris, J. R., \& Todaro, M. P. (1970). Migration, unemployment and development: a two-sector analysis. The American economic review, 60(1), 126-142.

Hart, K. (1970). 8. Small-scale entrepreneurs in Ghana and development planning. The Journal of Development Studies, 6(4), 104-120.

Instituto Nacional de Estadística, Geografía e Informática (INEGI). (2004). Anuario Estadístico de los Estados Unidos Mexicanos.

Jusidman, C. (1997). La informalidad en México: características y tendencias. En Memoria del III Seminario de Investigación Laboral: El sector informal urbano, STyPS, México.

Klein, E., y Tokman E. V. (Comps.). (1979). El subempleo en América Latina. Argentina: CLACSO, El Cid Editor.

Lewis, W. A. (1954). Economic development with unlimited supplies of labour. The manchester school, 22(2), 139-191.

OCDE. (2007). Manual sobre la medición de la economía no observada. ANDESTAD, Proyecto de Cooperación UE-CAN Estadísticas. Unión Europea, Comunidad Andina y OCDE. Secretaría General de la Comunidad Andina, Lima, Perú.

Organización Internacional del Trabajo - OIT. (1972). Employment, incomes and equality. A strategy for increasing productive employment in Kenya. Ginebra, OIT.

Organización Internacional del Trabajo - OIT. (1992). $79^{a}$ Conferencia Internacional del Trabajo, Mecanismos de fijación y control. Ginebra, OIT.

Organización Internacional del Trabajo - OIT. (1993). Resolución sobre la Clasificación Internacional de la Situación en el Empleo (CISE). Adoptada por la Decimoquinta Conferencia Internacional de Estadísticos del Trabajo. 
Organización Internacional del Trabajo - OIT. (1998). Resolución concerniente a la medición del subempleo y las situaciones de empleo inadecuado, adoptada por la decimosexta Conferencia Internacional de Estadísticos del Trabajo. Recuperada de https://www.ilo.org/public/spanish/bureau/stat/download/res/underemp.pdf

Organización Internacional del Trabajo - OIT. (2000). Empleo y protección social en el sector informal.

Organización Internacional del Trabajo - OIT. (2001). De la informalidad a la modernidad. Dirigido por Tokman E. Víctor

Organización Internacional del Trabajo - OIT. (2002). Informe VI de la 90 a Reunión de la Conferencia Internacional del Trabajo, OIT. Ginebra, 3-20 de junio de 2002. El trabajo decente y la economía informal.

Organización Internacional del Trabajo - OIT. (2003). Directrices sobre una definición estadística de empleo informal. Adoptadas por la Decimoséptima Conferencia Internacional de Estadísticos del Trabajo (noviembre - diciembre de 2003).

Organización Internacional del Trabajo - OIT. (2003). Decimoséptima Conferencia Internacional de Estadísticos del Trabajo. OIT. Ginebra.

Organización Internacional del Trabajo - OIT. (2005). Economía informal, Trabajo no declarado y Administración del trabajo. José Luis Daza. Departamento de Diálogo Social, Legislación y Administración del Trabajo. OIT. Ginebra.

Organización Internacional del Trabajo - OIT. (2008). Resolución sobre la medición del tiempo de trabajo. Decimoctava Conferencia Internacional de Estadísticos de Trabajo.

Organización Internacional del Trabajo - OIT. (2009). Guía sobre los nuevos indicadores de empleo de los objetivos de desarrollo del milenio: incluido el conjunto completo de indicadores de trabajo decente. Oficina Internacional del Trabajo. Ginebra. Recuperado de https://www.ilo.org/wcmsp5/groups/public/--ed_emp/documents/publication/wcms_110513.pdf

Organización Internacional del Trabajo - OIT. (2011a). Convenio sobre el trabajo decente para las trabajadoras y los trabajadores domésticos.

Organización Internacional del Trabajo - OIT. (2011b). Políticas y regulaciones para luchar contra el empleo precario. Oficina de Actividades para los Trabajadores (ACTRAV). Recuperado de http://www.ilo.org/wcmsp5/groups/public/---ed dialogue/--actrav/documents/meetingdocument/wcms_164288.pdf

Organización Internacional del Trabajo - OIT. (2012a). Del trabajo precario al trabajo decente. Documento final del Simposio de los Trabajadores sobre Políticas de Reglamentación para Luchar contra el Empleo Precario. 
Organización Internacional del Trabajo - OIT. (2012b). Medición de la economía informal, OIT. Ginebra. Recuperado de https://www.ilo.org/wcmsp5/groups/ public/---ed_emp/---emp_policy/documents/publication/wcms_229450.pdf

Organización Internacional del Trabajo - OIT. (2013a). Resolución sobre las estadísticas del trabajo, la ocupación y la subutilización de la fuerza de trabajo. $19^{a}$ Conferencia Internacional de Estadísticos del Trabajo, OIT.

Organización Internacional del Trabajo - OIT. (2013b). La medición de la informalidad: Manual estadistico sobre el sector informal y el empleo informal. OIT. Ginebra. Recuperado de https://www.ilo.org/wcmsp5/groups/public/--dgreports/---dcomm/---publ/documents/publication/wcms_222986.pdf

Organización Internacional del Trabajo - OIT. (2016). Protección social del trabajo doméstico. Tendencias y estadísticas. Documentos de política de protección social $N^{o} 16$. Organización Internacional del Trabajo. ILOSTAT.

Portes, A. (1995). En torno a la informalidad: ensayos sobre teoría y medición de la economía no regulada. México: Flacso.

Rossini, R. G., \& Thomas, J. J. (1988). Los fundamentos estadísticos de El otro sendero. Revista Desarrollo y Sociedad (21), 55-78.

Roubaud, F. (1995). La economía informal en México. ORSTOM, INEGI, Fondo de Cultura Económica.

Sistema de Cuentas Nacionales. (1993). Banco Mundial. Washington, D.C.

Soto, H. de. (1986). El otro sendero. Bogotá: Oveja Negra.

Tokman, E. V. (comp.). (1991). El sector informal en América Latina. Dos décadas de análisis. México.

Tokman, V.E., \& Klein, E. (1996). Regulation and the informal economy, microenterprises in Chile, Ecuador and Jamaica. Nueva York: Boulder, Lynne Rienner Publishers.

Vega, C. A., y Kruijt, D. (2000). El significado del sector informal y la microempresa en América Latina y en México. En Calvo, T., y Méndez, B. Micro y pequeña empresa en México. 